\title{
Lower prevalence of tuberculosis infection in BCG vaccinees: a cross-sectional study in adult prison inmates
}

\author{
Pei-Chun Chan, ${ }^{1,2,3}$ Chin-Hui Yang, ${ }^{1}$ Luan-Yin Chang, ${ }^{3}$ Kwei-Feng Wang, ${ }^{1}$ Yi-Chen Kuo, ${ }^{4}$ \\ Chou-Jui Lin, ${ }^{5}$ Shih-Wei Lee, ${ }^{5}$ Po-Ren Hsueh, ${ }^{6,7}$ Chi-Tai Fang, $^{2,7}$ Li-Min Huang $^{3}$
}

\begin{abstract}
- Additional supplementary files are published online only. To view these files please visit the journal online (http://dx. doi.org/10.1136/thoraxjn2012-202208).

${ }^{1}$ Third Division, Centers for Disease Control, Taipei, Taiwan ${ }^{2}$ Institute of Epidemiology and Preventive Medicine, College of Public Health, National Taiwan University, Taipei, Taiwan ${ }^{3}$ Department of Pediatrics, National Taiwan University Hospital, National Taiwan University College of Medicine, Taipei, Taiwan

${ }^{4}$ Guishan Township Health Station, Taoyuan County, Taiwan

${ }^{5}$ Department of Internal Medicine, Tao-Yuan General Hospital, Taipei, Taiwan ${ }^{6}$ Departments of Laboratory Medicine, National Taiwan University Hospital, National Taiwan University College of Medicine, Taipei, Taiwan ${ }^{7}$ Department of Internal Medicine, National Taiwan University Hospital, National Taiwan University College of Medicine, Taipei, Taiwan
\end{abstract}

\section{Correspondence to}

Dr Chi-Tai Fang, Institute of Epidemiology and Preventive Medicine, National Taiwan University, 17 Xu-Zhou Road, Taipei 100, Taiwan; fangct@ntu.edu.tw Dr. Li-Min Huang, Department of Pediatrics, National Taiwan University Hospital, 7 Chung-Shan South Road, Taipei 100, Taiwan; Imhuang@ntu.edu.tw

Received 28 May 2012 Revised 15 August 2012 Accepted 23 August 2012 Published Online First 27 September 2012

To cite: Chan P-C, Yang C-H, Chang $\mathrm{L}-\mathrm{Y}$, et al. Thorax 2013;68:263-268.

\section{ABSTRACT}

Objectives To address whether the effect of BCG vaccination against tuberculosis (TB) infection lasts to adulthood.

Methods A cross-sectional study on the prevalence of latent TB infection (LTBI) among HIV-negative men, using QuantiFERON-TB Gold In-tube (QFT-IT), was conducted at a prison in northern Taiwan with $>3000$ inmates. A QFT-IT $\geq 0.35 \mathrm{IU} / \mathrm{ml}$ was defined as LTBI. A QFT-IT $\geq 0.7 \mathrm{IU} / \mathrm{ml}$ was defined as recent LTBI. The association between the number of BCG scars and LTBI stratified by age was analysed. The study procedure was approved by the institutional review board, and all participants gave written informed consent before receiving screening tests.

Results Among the 2385 participants, 25\% had a QFT-IT $\geq 0.35 \mathrm{IU} / \mathrm{ml}$. Increasing LTBI (14\%, 32\% and $50 \%$ ) was observed with increased age (18-34 years, $35-54$ years and $\geq 55$ years) ( $p<0.001$ by the CochranArmitage Trend Test). The number of BCG scars were found to be inversely correlated with QFT-IT results for both $L T B I$ and recent $L T B I$ in all three age groups ( $p<0.001$ by Cochran-Mantel-Haenszel statistics). Conclusions Our results suggest that BCG vaccine seems to have a protective effect in adults decades after vaccination according to the number of recent infections (QFT-IT $\geq 0.7 \mathrm{IU} / \mathrm{ml}$ ). This finding has important implications for national policy of BCG vaccination. Further prospective cohort studies on the protective effect of BCG vaccination against TB infection in adults are warranted.

\section{INTRODUCTION}

The Bacillus Calmette-Guerin (BCG) vaccine was developed 90 years ago. ${ }^{1}$ BCG vaccine has a documented protective effect against tuberculosis (TB) meningitis and disseminated TB in children, and is used as a complementary strategy for TB control. $^{2} 3$ Greater than $80 \%$ of neonates and infants are covered by BCG in countries where the vaccine is part of the national childhood immunisation programme. ${ }^{4}$

However, the protective effect of BCG against pulmonary TB in previous studies remains controversial. A long-term follow-up study in Native Americans and two studies of cohorts in Brazil and the UK demonstrated $48 \%-59 \%$ protection against TB from BCG vaccine 10-50 years after vaccination in newborns, schoolchildren and the
Key messages

What is the key question?

- Does the effectiveness of BCG vaccine on latent TB infection last into adulthood?

What is the bottom line?

- There is an inverse correlation between the number of BCG scars and the prevalence of positive QFT-IT in all three age groups (18-34, $35-54$ and $\geq 55$ years). which indicates a lasting protective effect of BCG against TB infection into adulthood.

\section{Why read on?}

- This finding has important implications for the national policy of BCG vaccination.

community. ${ }^{5-7}$ Nevertheless, no evidence of substantial protection against TB from BCG vaccine was observed among schoolchildren and young adults in several randomised, controlled trials in the US State of Georgia and in Chennai, South India. ${ }^{8} 9$ Environmental mycobacteria exposure, differences between BCG strains used, genetic susceptibility and several other factors have been proposed as possible reasons for variable efficacy. ${ }^{1}{ }^{10}$

In immunocompetent individuals, $\mathrm{TB}$ infection often has an initial latent phase, in which the spread of TB bacilli is contained by the immune system and the subjects remain asymptomatic. ${ }^{11}$ In these patients, progression to symptomatic $\mathrm{TB}$ disease occurs only after the breakdown of this containment. ${ }^{12}$ Measuring BCG impact on latent TB infection (LTBI) may provide insight on the protective effect of BCG against the initial stage of TB pathogenesis.

Before the development of the interferon gamma release assay (IGRA) in early 2000, the tuberculin skin test (TST) was the only diagnostic tool for LTBI. Nevertheless, BCG vaccine per se can yield a false positive TST that complicates interpretations as evidenced by the discordance between TST and IGRA in persons who had received BCG. ${ }^{13}$ The arrival of IGRA provides a specific diagnostic tool for LTBI in BCG vaccinees. ${ }^{14}$ IGRA was used as an indicator for LTBI in children in Turkey in a 2005 study that demonstrated a $40 \%$ efficacy of BCG 
against LTBI. ${ }^{15}$ One outbreak investigation in a nursery for 2- to 5 -year-old children in the UK revealed that BCG vaccination provided $66 \%$ protection from infection. ${ }^{16}$ Does the same protective effect against LTBI from BCG vaccination also hold true in adults who received BCG vaccine after infancy? How long can BCG protect vaccinated individuals from LTBI? There are currently no answers for these questions.

The first BCG used in Taiwan was a liquid vaccine from an old Pasteur strain, which was certified by WHO and provided to TST-negative schoolchildren after $1953 .{ }^{17}$ BCG vaccine was subsequently changed, first to a new Pasteur strain in 1956 and then to a freeze-dried vaccine manufactured from the Japan Tokyo 172 strain in 1979. Since 1965, BCG has been provided to all infants and TST-negative schoolchildren. ${ }^{17}$ Revaccination of schoolchildren was halted in 1997 per WHO recommendation. ${ }^{18}$

To survey the prevalence of LTBI and its risk factors in adult prison inmates, a group vulnerable to $\mathrm{TB}$ transmission, the Centers for Disease Control, Taiwan (Taiwan CDC) provided a voluntary and free LTBI screening programme (including both TST and IGRA) to inmates at a prison in northern Taiwan. ${ }^{19}$ Among the inmates who agreed to participate, we analysed the effects of BCG vaccination on the prevalence of LTBI. We hypothesise that if BCG protects vaccinated individuals from TB infection, then in a cross-sectional LTBI survey stratified by age groups, we should observe an inverse association between the number of BCG inoculations and the prevalence of LTBI as determined by IGRA.

\section{MATERIALS AND METHODS Study setting}

Participants were enrolled from April to October 2008, from a prison near Taipei, Taiwan, that is capable of accommodating 3000 adult male inmates. The turnover of inmates in and out of the prison is high. Up to 300 new inmates arrive at this prison every 4 weeks.

\section{Ethics}

The institutional review board of the National Taiwan University Hospital reviewed and approved the study protocol (No. 200707047M). The screening was not compulsory. All participants gave written informed consent before receiving screening tests. Any active TB cases identified in this study were offered appropriate anti-TB drug therapy. Participants found to have LTBI by this study were also offered the option to receive LTBI treatment. ${ }^{19}$

\section{Eligibility}

HIV-negative inmates without active TB who understood the screening programme and who gave informed consent were enrolled. HIV-positive inmates were enrolled in another study. The exclusion criteria were as follows: inmates who had a prison term less than 6 months, who had received complete treatment for TB, or who had evidence based on a chest radiograph suggesting active TB.

\section{Evaluation for active TB disease}

All inmates were routinely offered a chest radiography evaluation and HIV testing upon their arrival at the prison. The frequency of chest radiography screening for new inmates was once per month. ${ }^{20}$ Inmates who had not yet completed screening radiographies were isolated from the other inmates. Sputum cultures for mycobacteria were used to exclude active TB when there were suspicious chest radiography findings or symptoms.
Chest radiography was provided to all participants who decided to receive the screening in our study, and the procedure of excluding active TB diseases was the same as the above process of sputum collections.

\section{Evaluation for LTBI}

The TST with purified protein derivative (RT 232 TU, Statens Serum Institute, Copenhagen, Denmark) and the QuantiFERON-TB Gold In-tube test (QFT-IT, Cellestis Ltd., Carnegie, Victoria, Australia) were conducted with the flow of drawing blood first, followed by performance of TST. The transverse induration size of the TST was read by the nurses who administered the test between 48 and $72 \mathrm{~h}$ after administration. Experts from the TST training committee double-checked the reading result of both BCG scars and the TST as part of a biannual training programme. A TST $\geq 10 \mathrm{~mm}$ (according to the Taiwan guidelines of TB diagnosis and treatment) and a QFT-IT $\geq 0.35 \mathrm{IU} / \mathrm{ml}$ (according to the rules of the manufacturers) were defined as positive. ${ }^{21}$ When analysing the association between the number of BCG scars and the TB infection risk, we further defined cases of recently acquired LTBI (recent LTBI) using a cut-off point of QFT-IT $\geq 0.7 \mathrm{IU} / \mathrm{ml}$. After acquisition of LTBI, the risk for progression to active $\mathrm{TB}$ is the highest in the first 2 years. $^{22}$ In parallel, a higher level of IFN- $\gamma$ response corresponds to a higher risk for progression to active $\mathrm{TB},{ }^{23}{ }^{24}$ which indicates that a high IFN- $\gamma$ response may be used as a marker for recent LTBI in immunocompetent persons. We chose $0.7 \mathrm{IU} / \mathrm{ml}$ as the cut-off value for recent LTBI because studies on serial testing of healthcare workers showed $0.35-0.7 \mathrm{IU} / \mathrm{ml}$ to be an uncertainty zone for recent TB infection. ${ }^{25} 26$

\section{Number of BCG inoculations}

The number of BCG scars was counted immediately prior to administration of the TST by trained public health nurses. Because there was no record of BCG immunisation in this study, we used BCG number as a proxy for previous BCG vaccination.

\section{Statistical analysis}

We compared categorical data by using the Pearson $\chi^{2}$ or Fisher exact tests, as appropriate. When analysing the association between the number of BCG scars and the TB infection risk, $2 \times N$ tables were analysed by the Cochran-Armitage trend test. Age-stratified analysis for the number of BCG scars and the TB infection risk was summarised by Cochran-Mantel-Haenszel statistics. The $\kappa$ value and agreement were calculated to compare the concordance of TST (three cut-off values) and QFT-IT (LTBI and recent LTBI). All analyses were conducted by using SAS, V.9.1 software (SAS Institute, Cary, North Carolina, USA).

\section{Role of the funding source}

The sponsor of the study had no role in study design, data collection, data analysis, data interpretation or writing of the report. The corresponding authors have full access to all the data in the study and have final responsibility for the decision to submit for publication.

\section{RESULTS}

A total of 4136 inmates were invited to complete the questionnaires for enrolment. Of a total of $3312(80 \%)$ inmates who agreed to be enrolled in our study, 2425 received at least one LTBI screening test, and 2385 had QFT-IT, TST and BCG data available for analysis (see figure 1 and table 1 for details). 


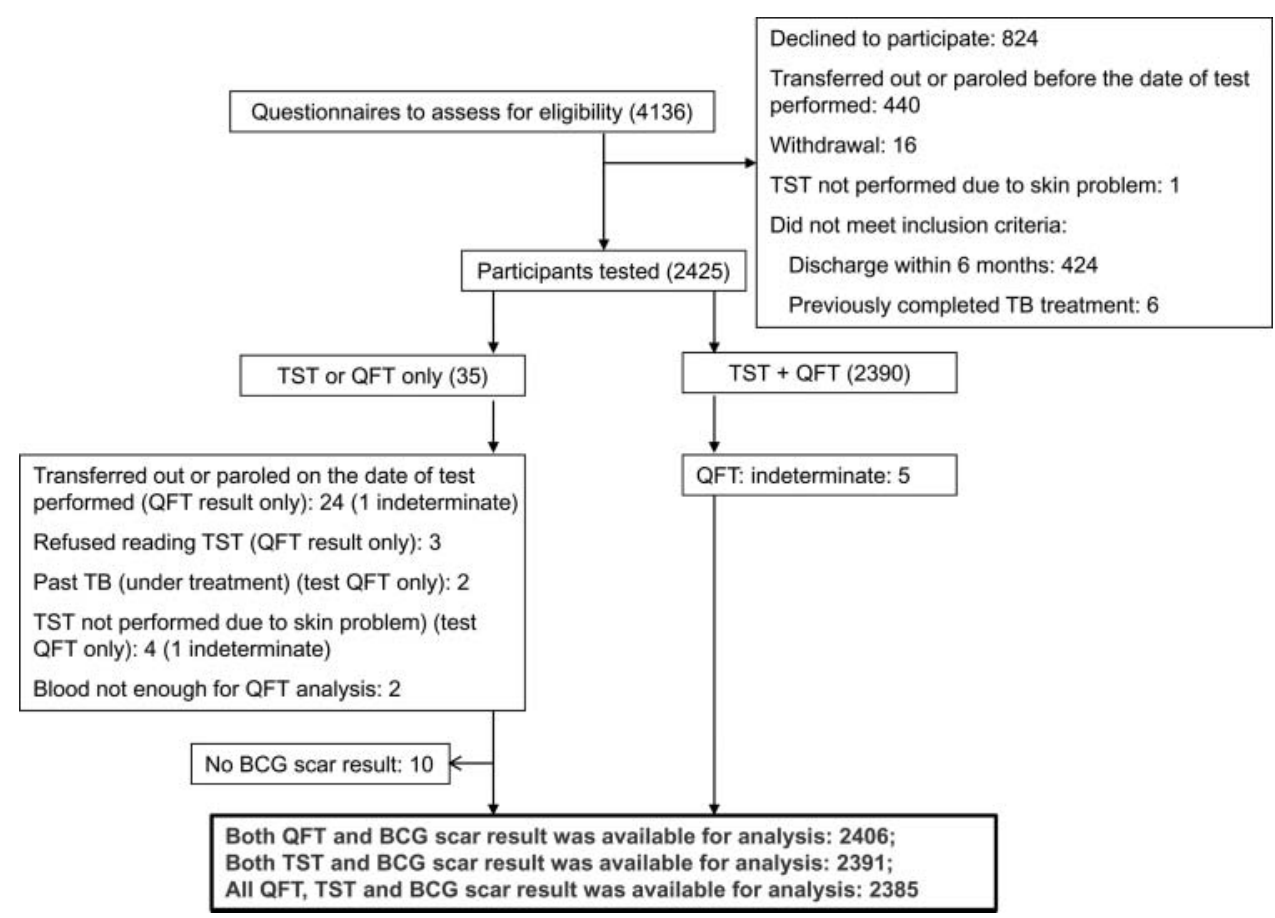

Figure 1 The flowchart of enrolment.

Overall, the QFT-IT was $\geq 0.35 \mathrm{IU} / \mathrm{ml}$ (LTBI) in $25 \%$ of the subjects and $\geq 0.7 \mathrm{IU} / \mathrm{ml}$ (recent LTBI) in $19 \%$ of the 2385 subjects included in the analysis. The TST was positive in $82 \%$ of

Table 1 Characteristics of participants who received at least one test in this study

\begin{tabular}{|c|c|c|c|c|}
\hline Variables & $\begin{array}{l}\text { Number of } \\
\text { persons }\end{array}$ & $\begin{array}{l}\text { Both results } \\
\text { available }\end{array}$ & $\begin{array}{l}\text { Only one } \\
\text { result } \\
\text { available }\end{array}$ & p Value ${ }^{*}$ \\
\hline & $\mathrm{N}(\%)$ & & & \\
\hline \multicolumn{5}{|l|}{ Age (years) } \\
\hline $18-24$ & $178(8)$ & $177(7)$ & $1(3)$ & 0.029 \\
\hline $25-34$ & $960(40)$ & $949(40)$ & $11(28)$ & \\
\hline $35-44$ & $754(31)$ & 741 (31) & $13(33)$ & \\
\hline $45-54$ & $389(16)$ & $381(16)$ & $8(20)$ & \\
\hline $55-64$ & $107(4)$ & $102(4)$ & $5(13)$ & \\
\hline$\geq 65$ & $37(2)$ & $35(1)$ & $2(5)$ & \\
\hline Total & $2425(100)$ & $2385(100)$ & $40(100)$ & \\
\hline \multicolumn{5}{|l|}{ Age (years) } \\
\hline $18-34$ & $1138(47)$ & $1126(47)$ & $12(30)$ & 0.003 \\
\hline $35-54$ & $1143(47)$ & $1122(47)$ & $21(53)$ & \\
\hline \multirow[t]{2}{*}{$\geq 55$} & $144(6)$ & $137(6)$ & $7(18)$ & \\
\hline & $2425(100)$ & $2385(100)$ & $40(100)$ & \\
\hline \multicolumn{5}{|l|}{ Body weight } \\
\hline$\geq 70 \mathrm{~kg}$ & $1229(51)$ & $1206(51)$ & $23(58)$ & 0.384 \\
\hline \multicolumn{5}{|l|}{ Prison term } \\
\hline$>6$ months & $2423(100)$ & $2383(100)$ & $40(100)$ & 1.0 \\
\hline \multicolumn{5}{|l|}{ Education } \\
\hline$\geq 13$ years & $1101(45)$ & $1079(45)$ & $22(55)$ & 0.219 \\
\hline \multicolumn{5}{|l|}{ BCG scar no } \\
\hline No inoculation & $218(9)$ & $212(9)$ & $17(43)$ & $<0.001$ \\
\hline Inoculation once & 1065 (44) & $1053(44)$ & $12(30)$ & \\
\hline \multirow[t]{2}{*}{ Booster } & 1131 (47) & 1120 (47) & $11(28)$ & \\
\hline & $2414(100)$ & & & \\
\hline
\end{tabular}

the subjects with a cut-off point of $10 \mathrm{~mm}, 45 \%$ of the subjects with a cut-off of $15 \mathrm{~mm}$, and $17 \%$ of the subjects with a cut-off of $18 \mathrm{~mm}$. The concordance of these two tests was analysed according to different cut-offs for both the TST and QFT-IT (table 2). The $\kappa$ values were 0.09 for a TST $\geq 10 \mathrm{~mm}$ and a QFT-IT $\geq 0.35 \mathrm{IU} / \mathrm{ml}$, indicating a poor concordance between the two tests. The $\kappa$ values were up to 0.243 for a TST $\geq 18 \mathrm{~mm}$ and a QFT-IT $\geq 0.7 \mathrm{IU} / \mathrm{ml}$.

Tables 3 and online Appendix table 2 show the positive rate of QFT-IT and TST, stratified by age groups and the number of BCG scars. Increasing positivity of QFT-IT was observed with increased age $(p<0.001$ by the Cochran-Armitage Trend Test). QFT-IT results were found to be inversely correlated with the number of BCG scars when two different cut-offs were used (LTBI or recent LTBI) in all three age groups $(p<0.001$ by Cochran-Mantel-Haenszel statistics) (table 3). By contrast, the TST positivity increased with the number of BCG scars when a cut-off of $10 \mathrm{~mm}$ was used $(\mathrm{p}<0.001)$, but the trend was not observed when cut-offs of $15 \mathrm{~mm}$ or $18 \mathrm{~mm}$ were used (appendix table S2).

The evaluation for active TB disease identified only one case. The individual was asymptomatic at the time of arrival, but the chest radiograph showed a suspicious pulmonary lesion during the entry screening. However, 2 months later, the individual was determined as not having active TB because his cultures were negative and his chest radiography was stable. He therefore received an LTBI test, and the double-positive result was confirmed. He did not receive LTBI treatment. Two years later, bacteriology determined that the individual had active TB.

\section{DISCUSSION}

Our results showed that after stratification by age, there was an inverse correlation between the number of BCG scars and the prevalence of positive QFT-IT, which indicates a protective effect of BCG against TB infection. Because the association was significant in all age groups (18-34 years, 35-54 years and 
Table 2 Summary statistics for the double positive results (QuantiFERON-TB Gold In-tube and TST) and the analysis for concordance between the two tests

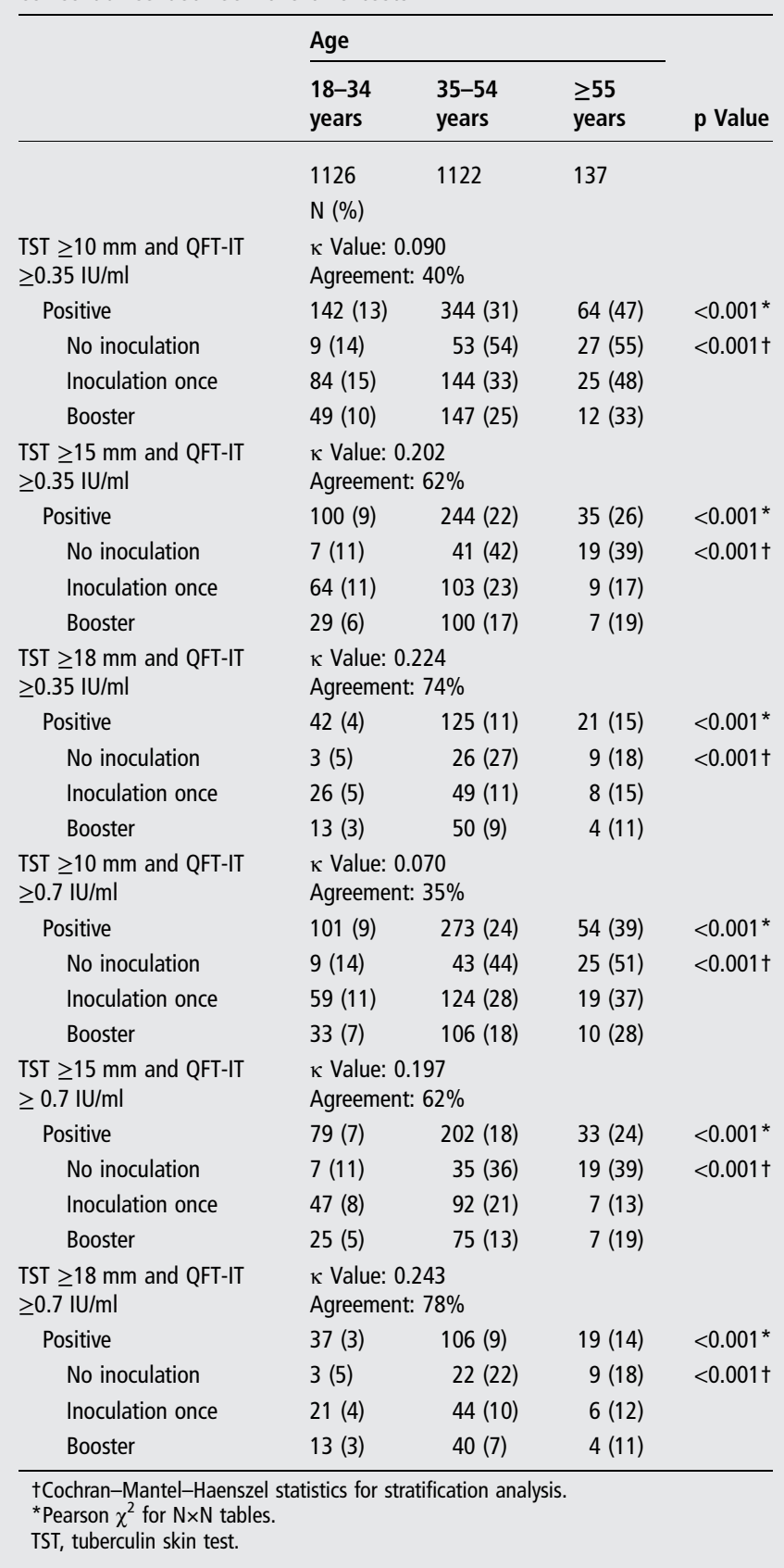

$\geq 55$ years), our results suggest that BCG vaccine seems to have a protective effect in adults decades after vaccination.

Our observation that BCG vaccination may provide protection against $\mathrm{TB}$ infection in adults is consistent with the previously reported findings in Turkey and the UK that there is a higher LTBI rate in unvaccinated children compared with those who were vaccinated. ${ }^{15}{ }^{16}$ Diel et al also reported some evidence supporting a protective effect from BGC vaccination in 104 children aged < 16 years in a cohort in Hamburg, Germany. ${ }^{24}$ The QFT-IT positive rate was higher in unvaccinated children $(26.5 \%)$ than in those who were vaccinated (13.9\%), although this difference did not reach statistical significance due to the small sample size.

Whether revaccination in adolescents or adults is beneficial or useless in TB control has long been a subject of debate.
Table 3 Summary statistics for results of QuantiFERON-TB Gold In-tube

\begin{tabular}{|c|c|c|c|}
\hline Stratification & $\begin{array}{l}\text { Number of } \\
\text { persons }\end{array}$ & $\begin{array}{l}\text { Cut-off for } \\
\text { positivity (\%) }\end{array}$ & $\begin{array}{l}\text { Cut-off for } \\
\text { positivity (\%) }\end{array}$ \\
\hline & 2416 & $\begin{array}{l}\text { LTBI (QFT-IT } \\
\geq 0.35 \mathrm{IU} / \mathrm{ml})\end{array}$ & $\begin{array}{l}\text { Recent LTBI } \\
\text { (QFT-IT } \\
\geq 0.7 \mathrm{IU} / \mathrm{ml})\end{array}$ \\
\hline Age (years) & & $\mathrm{N}(\%)$ & \\
\hline $18-24$ & 178 & $15(8)$ & $12(7)$ \\
\hline $25-34$ & 955 & $141(15)$ & $98(10)$ \\
\hline $35-44$ & 753 & $205(27)$ & $161(21)$ \\
\hline $45-54$ & 389 & $163(42)$ & $130(33)$ \\
\hline $55-64$ & 105 & $50(48)$ & $41(39)$ \\
\hline$\geq 65$ & 36 & $20(56)$ & $18(50)$ \\
\hline$\chi^{2} p$ Value & & $<0.001$ & $<0.001$ \\
\hline \multicolumn{4}{|l|}{ Age (years) } \\
\hline $18-34$ & 1133 & $156(14)$ & $110(10)$ \\
\hline $35-54$ & 1142 & $368(32)$ & $291(25)$ \\
\hline \multirow[t]{2}{*}{$\geq 55$} & 141 & $70(50)$ & $59(42)$ \\
\hline & & $<0.001^{*}$ & $<0.001^{*}$ \\
\hline BCG scar number & 2406 & $\mathrm{~N}(\%)$ & \\
\hline No inoculation & 216 & $102(47)$ & $86(40)$ \\
\hline Inoculation once & 1062 & $271(26)$ & $216(20)$ \\
\hline \multirow[t]{2}{*}{ Booster } & 1128 & $218(19)$ & $155(14)$ \\
\hline & & $<0.001^{*}$ & $<0.001^{*}$ \\
\hline Controlling for age & & $<0.001 \dagger$ & $<0.001 \dagger$ \\
\hline 18-34 years & & $\mathrm{N}(\%)$ & \\
\hline No inoculation & 65 & $12(18)$ & $10(15)$ \\
\hline Inoculation once & 563 & $93(17)$ & $66(12)$ \\
\hline \multirow[t]{2}{*}{ Booster } & 504 & $51(10)$ & $34(7)$ \\
\hline & & $p=0.002^{*}$ & $p=0.002^{*}$ \\
\hline \multicolumn{4}{|l|}{$35-54$ years } \\
\hline No inoculation & 101 & $60(59)$ & $48(48)$ \\
\hline Inoculation once & 446 & $150(34)$ & $129(29)$ \\
\hline \multirow[t]{2}{*}{ Booster } & 587 & $155(26)$ & $111(19)$ \\
\hline & & $p<0.001^{*}$ & $p<0.001^{*}$ \\
\hline \multicolumn{4}{|l|}{$\geq 55$ years } \\
\hline No inoculation & 50 & $30(60)$ & $28(56)$ \\
\hline Inoculation once & 53 & $28(53)$ & $21(40)$ \\
\hline \multirow[t]{2}{*}{ Booster } & 37 & $12(32)$ & $10(27)$ \\
\hline & & $p=0.013^{*}$ & $p=0.006^{*}$ \\
\hline
\end{tabular}

A double-blind, randomised, placebo-controlled trial conducted in Karonga, Malawi, between 1986 and 1987 showed that revaccination with BCG did not provide any protection against $\mathrm{TB}$, but that primary vaccination or revaccination did provide protection against leprosy. ${ }^{27} \mathrm{WHO}$ officially recommended to not revaccinate with BCG in $1995,{ }^{18}$ and Taiwan followed the recommendation and stopped the revaccination programme in $1997 .{ }^{17}$ However, we should note that the Karonga trial in Malawi that documented the failed BCG revaccination strategy also failed to find primary BCG vaccination protective against TB. ${ }^{10}$ A retrospective cohort consisting of 303692 children born between 1978 and 1982 in Hong Kong who received the primary BCG in infancy and a booster at 6-9 years of age also failed to demonstrate any significant difference in the TB rate 
between participants and non-participants in a school revaccination programme. ${ }^{28}$ An open-label, no-placebo, clustered, randomised, controlled trial was conducted among 200805 schoolchildren aged 7-14 years in Salvador and Manaus, Brazil, 5 years later. ${ }^{29}$ In the initial follow-up for 3-4 years, no substantial additional protection from BCG revaccination in schoolchildren was found. However, after 9 years of follow-up, a 33\% $(3 \%-54 \%)$ vaccine efficacy in children aged $<11$ years was reported recently in Salvador, a location with a lower nontuberculous mycobacteria (NTM) prevalence. ${ }^{30}$ This finding was comparable with the $30 \%(3 \%-52 \%)$ protective effect from BCG revaccination observed in non-reactors to NTM, 15 years after a double-blind, randomised, controlled trial conducted in South India. ${ }^{31}$ The inverse dose response of BCG vaccine over the TB infection risk noted in our study further supports the possibility that revaccination may have some protective effect.

The inconsistent protective effect from either BCG vaccination or revaccination has caused a number of debates over the past two decades. ${ }^{1} 10$ The difference in antigenicity between BCG strains and the epidemic strains prevalent in some regions could be factors behind the discrepancies between studies conducted in different geographical regions. Theoretically, the heavy use of BCG in high-burden regions could also drive the evolution of prevalent Mycobacterium tuberculosis strains. This hypothesis was proposed because of a case-control study of TB patients conducted in Vietnam, Hong Kong, China and The Netherlands, which revealed that a higher proportion of BCG-vaccinated TB cases were infected with the modern Beijing strain of $M$ tuberculosis after age stratification. ${ }^{32}$ Further studies using cohort or randomised control trial designs to compare the protective efficacy of BCG against Beijing strains versus non-Beijing strains are required to clarify these important issues.

Consistent with previous reports from Korea, which has 95.7\% BCG coverage and moderate LTBI risk, ${ }^{33}$ our results showed only an unsatisfactory concordance between TST and QFT. Similarly, discordance between TSTs and IGRAs was observed in Japanese health workers who had predominately undergone multiple BCG vaccinations. ${ }^{34}$ The concordance between the TST and QFT was also low in BCG-vaccinated Korean healthcare workers $(\kappa=0.31$, agreement $67.5 \%$ ). ${ }^{35}$ The high false positive rates of the TST after BCG vaccination has raised concerns of unnecessary LTBI diagnosis and treatment, ${ }^{36} 37$ and can explain why the inverse correlation between BCG vaccination and LTBI could not be discovered by previous TST-based studies.

Similar to many previous studies, ${ }^{15} 38$ we used the number of BCG scars as a proxy for BCG vaccination record. The proportion of vaccinated people who develop and retain a recognisable scar following vaccination has been reported to vary from $98.9 \%$, in a vaccine trial in South India 4 years after vaccination, ${ }^{38}$ to $60 \%$ in Swedish children assessed 14 years after vaccination at birth. ${ }^{39}$ In the present study, nurses did not know the result of LTBI when they counted the number of BCG scars. Therefore, any misclassification of a BCG scar would very likely be non-differential and yield a bias toward null. Our estimate for the correlation of more BCG scars with a lower rate of LTBI is, therefore, a conservative one.

In the present study, the lower prevalence of LTBI in BCG vaccinees is unlikely to be confounded by social economic status in early life because (1) BCG vaccination is free in Taiwan and (2) BCG vaccination is universally provided through public health stations (newborn and school children), hospitals (newborn), and schools (school children) in Taiwan. Thus, families from all social classes have the same access to BCG vaccination.
Our study has some limitations. Although up to $80 \%$ (3312/4136) of inmates were willing to be enrolled, 13\% (440/3312) of them could not receive the test due to transfer or parole. As the age categories in stratification are broad (spanning 20 years), the relationship between BCG vaccination and LTBI could be confounded by a birth cohort effect if the TB trends changed rapidly over time. However, the nationwide TB registry data in Taiwan over the past half century (appendix table S1) show no such change, which makes confounding by birth cohort effect unlikely. Because of the limitations in the design of the cross-sectional study, the temporality between a lack of BCG vaccination and the occurrence of LTBI in participants is unclear. Furthermore, we were not informed of the reasons for the different numbers of vaccinations (or the lack of vaccination) among the participants. Therefore, factors influencing whether BCG has been received during infancy or childhood could confound our results. Nevertheless, analysis using QFT-IT $\geq 0.7 \mathrm{IU} / \mathrm{ml}$ as a cut-off value, a marker of recently acquired LTBI, also yielded the same result, indicating that the observed effect is genuine rather than confounded or a coincidence. Therefore, our results justify further prospective cohort studies to clarify the causal relationship between BCG vaccine and protection against LTBI.

In conclusion, our results suggest that BCG vaccine seems to have a protective effect in adults decades after vaccination according to the number of recent infections (QFT-IT $\geq 0.7 \mathrm{IU} / \mathrm{ml}$ ). This finding has important implications for the continuation of a national policy of BCG vaccination. Further prospective cohort studies on the protective effect of BCG vaccination against TB infection in adults are warranted.

Acknowledgements We thank all the participants who took part in the study, and the employees and physicians in Taipei Prison, Guishan Township, Taoyuan County. We acknowledge the crucial support of the Third Division, Taiwan CDC. We thank all the public health nurses who conducted the standard operational procedures for TST injection and reading in the study.

Contributors PCC, CHY, LYC, KFW, PRH, LMH: designed the study; PCC, CHY LYC, KFW, YCK, CJL, SWL, PRH, LMH: conducted the study; PCC: performed the statistical analysis; PCC, CTF: wrote the first draft of the manuscript, with all authors contributing to the final draft; all authors contributed to the data interpretation and critically reviewed the manuscript; CTF, LMH: contributed equally to the study.

Funding This study was supported by the Centers for Disease Control, Taiwan (Grant DOH97-DC-1502), to which interim progress reports were submitted. The funding body had no role in study design, data analysis, data interpretation or writing of the report, except data collection.

Competing interests All authors have completed the Unified Competing Interest form at www.icmje.org/coi_disclosure.pdf (available on request from the corresponding author) and declare: no support from any organisation for the submitted work; no financial relationships with any organisations that might have an interest in the submitted work in the previous 3 years; no other relationships or activities that could appear to have influenced the submitted work.

Ethics approval The institutional review board of the National Taiwan University Hospital reviewed and approved the study protocol (No. 200707047M).

Provenance and peer review Not commissioned; externally peer reviewed.

Data sharing statement The dataset is available from the corresponding author at pcanita.tw@cdc.gov.tw.

\section{REFERENCES}

1 Fine PEM, Carneiro IAM, Milstein JB, et al. Issues relating to the use of BCG in immunization programs. Geneva: World Health Organization, 1999.

2 Rodrigues LC, Diwan VK, Wheeler JG. Protective effect of BCG against tuberculous meningitis and miliary tuberculosis: a meta-analysis. Int J Epidemiol 1993;22:1154-8.

3 Colditz GA, Berkey CS, Mosteller F, et al The efficacy of bacillus Calmette-Guerin vaccination of newborns and infants in the prevention of tuberculosis: meta-analyses of the published literature. Pediatrics 1995;96:29-35.

4 World Health Organization. BCG vaccine WHO positional paper. Wkly Epidemiol Rec 2004; 79:25-40 
5 Aronson NE, Santosham M, Comstock GW, et al. Long-term efficacy of BCG vaccine in American Indians and Alaska natives a 60-year follow-up study. JAMA 2004:291:2086-91.

6 Barreto ML, Cunha SS, Pereira SM, et al. Neonatal BCG protection against tuberculosis lasts for 20 years in Brazil. Int J Tuberc Lung Dis 2005:9:1171-3.

7 Hart PD, Sutherland I. BCG and vole bacillus vaccines in the prevention of tuberculosis in adolescence and early adult life. BMJ 1977;2:293-5.

8 Comstock GW, Webster RG. Tuberculosis studies in Muscogee County, Georgia, VII. A twenty year evaluation of BCG vaccination in school population. Am Rev Respir Dis 1969:100:839-45.

9 Tuberculosis Prevention Trial, Madras. Trial of BCG vaccines in south India for tuberculosis prevention: first report. Bull World Health Organ 1979:57:819-27.

10 Smith D, Wiegeshaus E, Balasubramanian V. An analysis of some hypotheses related to the Chingelput bacille Calmette-Guérin trial. Clin Infect Dis 2000;31 (Suppl 3):S77-80.

11 Dietrich J, Doherty TM. Interaction of Mycobacterium tuberculosis with the host: consequences for vaccine development. APMIS 2009;117:440-57.

12 Russell DG, Barry CE, Flynn JL. Tuberculosis: what we don't know can, and does, hurt us. Science 2010;328:852-6.

13 Diel $R$, Goletti $D$, Ferrara $G$, , et al Interferon- $\gamma$ release assays for the diagnosis of latent Mycobacterium tuberculosis infection: a systematic review and meta-analysis. Eur Respir J 2011;37:88-99.

14 Pai M, Zwerling A, Menzies D. Systematic review: T-cell-based assays for the diagnosis of latent tuberculosis infection: an update. Ann Intern Med 2008;149:177-84.

15 Soysal A, Millington KA, Bakir M, et al Effect of BCG vaccination on risk of Mycobacterium tuberculosis infection in children with household tuberculosis contact: a prospective community-based study. Lancet 2005;366:1443-51.

16 Eriksen J, Chow JY, Mellis V, et al. Protective effect of BCG vaccination in a nursery outbreak in 2009: time to reconsider the vaccination threshold? Thorax 2010:65:1067-71.

17 Provincial Chronic Disease Control Bureau. A review of the tuberculosis control program in Taiwan, 1949-1989: Chronological Development of the Program, BCG vaccination. Taipei, Taiwan: Provincial Chronic Disease Control Bureau, 1991:4-37.

18 World Health Organization. Statement on BCG revaccination for the prevention of tuberculosis. Wkly Epidemiol Rec 1995;70:229-36.

19 Chan PC, Yang CH, Chang LY, et al. Latent tuberculosis infection treatment for prison inmates: a randomised controlled trial. Int J Tuberc Lung Dis 2012;16:633-8.

20 Chiang CY, Hsu CJ, Hsu PK, et al. Pulmonary tuberculosis in the Taiwanese prison population. J Formos Med Assoc 2002;101:537-41.

21 Centers for Disease Control, Department of Health. Taiwan guidelines for TB diagnosis \& treatment. 2nd edn. Taipei, Taiwan: CDC, 2006

22 Horsburgh CR Jr. Priorities for the treatment of latent tuberculosis infection in the United States. N Engl J Med 2004;350:2060-7.

23 Yoshiyama T, Harada N, Higuchi $\mathrm{K}$, et al. Use of the QuantiFERON-TB Gold test for screening tuberculosis contacts and predicting active disease. Int J Tuberc Lung Dis 2010;14:819-27.
24 Diel R, Loddenkemper R, Niemann $\mathrm{S}$, et al. Negative and positive predictive value of a whole-blood interferon-gamma release assay for developing active tuberculosis: an update. Am J Respir Crit Care Med 2011;183:88-95.

25 Costa JT, Rui S, Raul S, et al. Serial testing with the interferon-gamma release assay in Portuguese healthcare workers. Int Arch Occup Environ Health 2011;84:461-9.

26 Ringshausen FC, Schablon A, Nienhaus A. Interferon-gamma release assays for the tuberculosis serial testing of health care workers: a systematic review. J Occup Med Toxicol 2012;7:6.

27 Karonga Prevention Trial Group. Randomized controlled trial of single BCG, repeated BCG, or combined BCG and killed Mycobacterium leprae vaccine for prevention of leprosy and tuberculosis in Malawi. Lancet 1996:348:17-24.

28 Leung CC, Tam CM, Chan SL, et al. Efficacy of the BCG revaccination programme in a cohort given BCG vaccination at birth in Hong Kong. Int J Tuberc Lung Dis 2001;5:717-23.

29 Rodrigues LC, Pereira SM, Cunha SS, et al Effect of BCG revaccination on incidence of tuberculosis in school-aged children in Brazil: the BCG-REVAC cluster-randomised trial. Lancet 2005;366:1290-5.

30 Barreto ML, Pereira SM, Pilger D, et al Evidence of an effect of BCG revaccination on incidence of tuberculosis in school-aged children in Brazil: second report of the BCG-REVAC cluster-randomised trial. Vaccine 2011:29:4875-7.

31 Narayanan PR. Influence of sex, age \& nontuberculous infection at intake on the efficacy of BCG: re-analysis of 15-year data from a double-blind randomized control trial in South India. Indian J Med Res 2006;123:119-24.

32 Kremer K, van-der-Werf MJ, Au BK, et al Vaccine induced immunity circumvented by typical mycobacterium tuberculosis Beijing strains. Emerg Infect Dis 2009;15:335-9.

33 Kang YA, Lee HW, Yoon HI, et al. Discrepancy between the tuberculin skin test and the whole-blood interferon gamma assay for the diagnosis of latent tuberculosis infection in an intermediate tuberculosis-burden country. JAMA 2005;293:2756-61.

34 Harada N, Nakajima Y, Higuchi K, et al. Screening for tuberculosis infection using whole-blood interferon-gamma and Mantoux testing among Japanese healthcare workers. Infect Control Hosp Epidemiol 2006:27:442-8.

35 Choi JC, Shin JW, Kim JY, et al. The effect of previous tuberculin skin test on the follow-up examination of whole-blood interferon-gamma assay in the screening for latent tuberculosis infection. Chest 2008;133:1415-20.

36 Nienhaus A, Schablon A, Diel R. Interferon-gamma release assay for the diagnosis of latent TB infection-analysis of discordant results, when compared to the tuberculin skin test. PLOS ONE 2008;3:e2665.

37 O'Neal S, Hedberg K, Markum A, et al. Discordant tuberculin skin and interferon-gamma tests during contact investigations: a dilemma for tuberculosis controllers. Int J Tuberc Lung Dis 2009;13:662-4.

38 Tuberculosis Prevention Trial, Madras. Trial of BCG vaccines in south India for tuberculosis prevention. Indian J Med Res 1980:72:S1-74.

39 Beskow R, Bleiker M, Dahlstrom G, et al. Sensitivity to tuberculin in Swedish schoolboys vaccinated with BCG at birth. Bull Int Union Tuberc 1980;55:101-6. 